\title{
3D Variable Coefficient KdV Equation and Atmospheric Dipole Blocking
}

\author{
Juanjuan Ji $\left(\mathbb{D},{ }^{1,2}\right.$ Yecai Guo $\mathbb{D}^{1},{ }^{1}$ Lanfang Zhang, ${ }^{2}$ and Lihua Zhang ${ }^{2}$ \\ ${ }^{1}$ College of Atmospheric Science, School of Electronic and Information Engineering, \\ Nanjing University of Information Science and Technology, Nanjing, Jiangsu 210044, China \\ ${ }^{2}$ School of Physics and Electrical Engineering, Anqing Normal University, Anqing, Anhui 246133, China \\ Correspondence should be addressed to Juanjuan Ji; jjj0721@126.com; and Yecai Guo; guo-yecai@163.com
}

Received 4 January 2018; Revised 5 March 2018; Accepted 14 March 2018; Published 17 May 2018

Academic Editor: Herminia García Mozo

Copyright (C) 2018 Juanjuan Ji et al. This is an open access article distributed under the Creative Commons Attribution License, which permits unrestricted use, distribution, and reproduction in any medium, provided the original work is properly cited.

\begin{abstract}
A $(2+1)$-dimensional variable coefficient Korteweg-de Vries (3D VCKdV) equation is first derived in this paper by means of introducing 2-dimensional space and time slow-varying variables and the multiple-level approximation method from the wellknown barotropic and quasi-geostrophic potential vorticity equation without dissipation. The exact analytical solution of the 3D VCKdV equation is obtained successfully by making use of CK's direct method and the standard Zakharov-Kuznetsov equation. By some arbitrary functions and the analytical solution, a dipole blocking evolution process with twelve days' lifetime is described, and the result illustrates that the central axis of the dipole is no longer perpendicular to the vertical direction but has a certain angle to vertical direction. The comparisons with the previous researches and Urals dipole blocking event demonstrate that 3D VCKdV equation is more suitable for describing the complex atmospheric blocking phenomenon.
\end{abstract}

\section{Introduction}

Atmospheric blocking is a nonlinear phenomenon with a long lifetime occurring in mid-high latitude regions. Its dynamical study has been an important research topic in the atmospheric science field because of its significant influence on disaster weathers and extreme cold events. In the past decades, many investigators have proposed various nonlinear theories such as Rossby soliton [1-4], envelope Rossby soliton that is described by the Schrodinger equation $[5,6]$, and others to explain the formation of atmospheric blocking and its life process. Although the eddy-forced envelope soliton model can describe a life cycle of atmospheric dipole blocking $[7,8]$, the KdV-type soliton cannot represent the time variation or life of atmospheric dipole blocking [2,3]. However, by considering the time slow- varying basic flow, the KdV-type Rossby solitary wave can represent the life cycle of dipole blocking [9]. Previously derived constant coefficient KdV- and Schrödinger-type equations and variable coefficient $\mathrm{KdV}$ - and Schrödinger-type equations are all one-dimensional models on the space [10-15]. However, in the nature, propagation of a solitary wave is usually two-dimensional on the space, and only thinking about onedimensional model may not be enough.

The purpose of this paper is to extend the solitary Rossby wave model to the 3D case, namely, two-dimensional space and time. We aim to derive a $(2+1)$-dimensional variable coefficient KdV (3D VCKdV) equation from the barotropic and quasi-geostrophic potential vorticity equation without dissipation on a beta-plane.

The structure of this paper is as follows: the $3 \mathrm{D} \mathrm{VCKdV}$ equation is derived in Section 2; in Section 3, the exact analytical solution of the equation is obtained; Section 4 is devoted to study atmospheric dipole blocking by some arbitrary functions and parameters, and a comparison with the previous model and Urals dipole blocking is made. In the last section, some conclusions are given.

\section{Derivation of 3D VCKdV Equation}

The barotropic and quasi-geostrophic potential vorticity equation without dissipation on a beta-plane in the atmospheric dynamical system is as follows: 


$$
\left(\frac{\partial}{\partial t}+\frac{\partial \psi}{\partial x} \frac{\partial}{\partial y}-\frac{\partial \psi}{\partial y} \frac{\partial}{\partial x}\right)\left(\nabla^{2} \psi-\lambda_{0}^{2} \psi\right)+\beta_{0} \frac{\partial \psi}{\partial x}=0
$$

which is a highly nonlinear equation. It is very difficult to solve. In (1), $\psi$ is the stream function; $\beta_{0}=\left(\omega_{0} / R_{0}\right) / \cos \phi_{0}$, in which $R_{0}$ is the earth's radius, $\omega_{0}$ is the angular frequency of the earth's rotation, and $\phi_{0}$ is the latitude; $\lambda_{0}=f_{0} / \sqrt{g H}$, in which $f_{0}$ is the Coriolis parameter, $g$ is the gravitational acceleration, and $H$ is the atmospheric average height.

Let us assume that there is a base flow independent of variable $x$ in the atmospheric system; thus, the stream function is rewritten as

$$
\psi=\Psi_{0}(y, t)+\varepsilon^{2} \psi^{\prime},
$$

where $\psi^{\prime}$ is the perturbation stream function, $\varepsilon<1$ is a small parameter, and base flow field $\Psi_{0}(y, t)$ is a function of variables $y$ and $t$; in the previous studies, it is often taken only as a function of $y$. For simplicity of notation, the prime is dropped out in the remaining of this paper.

Substituting (2) into (1), we obtain

$$
\begin{aligned}
& \left(\frac{\partial}{\partial t}-\Psi_{0 y} \frac{\partial}{\partial x}\right) \nabla^{2} \psi+J\left(\psi, \nabla^{2} \psi\right)-\lambda_{0}^{2} \frac{\partial \psi}{\partial t} \\
& \quad+\left(\beta_{0}+\Psi_{0 y y y}\right) \frac{\partial \psi}{\partial x}-\left(\lambda_{0}^{2}-\frac{\partial^{2}}{\partial y^{2}}\right) \Psi_{0 t}=0 .
\end{aligned}
$$

Because of the multiple time-space scale features of the solitary wave in the fluid, we introduce 2-dimensional space and time slow-varying variables:

$$
\begin{aligned}
& X=\varepsilon\left(x-c_{0} t\right), \\
& Y=\varepsilon y, \\
& y=y, \\
& T=\varepsilon^{3} t,
\end{aligned}
$$

where $c_{0}$ is an arbitrary constant. From (4), we have

$$
\begin{aligned}
\frac{\partial}{\partial x} & =\varepsilon \frac{\partial}{\partial X}, \\
\frac{\partial^{2}}{\partial x^{2}} & =\varepsilon^{2} \frac{\partial^{2}}{\partial X^{2}}, \\
\frac{\partial}{\partial t} & =-c_{0} \varepsilon \frac{\partial}{\partial X}+\varepsilon^{3} \frac{\partial}{\partial T}, \\
\frac{\partial}{\partial y} & =\varepsilon \frac{\partial}{\partial Y}+\frac{\partial}{\partial y}, \\
\frac{\partial^{2}}{\partial y^{2}} & =\varepsilon^{2} \frac{\partial^{2}}{\partial Y^{2}}+2 \varepsilon \frac{\partial^{2}}{\partial y \partial Y}+\frac{\partial^{2}}{\partial y^{2}} .
\end{aligned}
$$

Consequently,

$$
\nabla^{2} \psi=\varepsilon^{2} \frac{\partial^{2} \psi}{\partial X^{2}}+\varepsilon^{2} \frac{\partial^{2} \psi}{\partial Y^{2}}+2 \varepsilon \frac{\partial^{2} \psi}{\partial y \partial Y}+\frac{\partial^{2} \psi}{\partial y^{2}},
$$

$$
\begin{aligned}
J\left(\psi, \nabla^{2} \psi\right)= & \frac{\partial \psi}{\partial x} \frac{\partial}{\partial y} \nabla^{2} \psi-\frac{\partial \psi}{\partial y} \frac{\partial}{\partial x} \nabla^{2} \psi \\
= & \varepsilon \frac{\partial \psi}{\partial X}\left[\varepsilon \frac{\partial}{\partial Y}\left(\varepsilon^{2} \frac{\partial^{2} \psi}{\partial X^{2}}+\varepsilon^{2} \frac{\partial^{2} \psi}{\partial Y^{2}}+2 \varepsilon \frac{\partial^{2} \psi}{\partial y \partial Y}+\frac{\partial^{2} \psi}{\partial y^{2}}\right)\right. \\
& \left.+\frac{\partial}{\partial y}\left(\varepsilon^{2} \frac{\partial^{2} \psi}{\partial X^{2}}+\varepsilon^{2} \frac{\partial^{2} \psi}{\partial Y^{2}}+2 \varepsilon \frac{\partial^{2} \psi}{\partial y \partial Y}+\frac{\partial^{2} \psi}{\partial y^{2}}\right)\right] \\
& -\varepsilon\left(\varepsilon \frac{\partial \psi}{\partial Y}+\frac{\partial \psi}{\partial y}\right) \frac{\partial}{\partial X}\left(\varepsilon^{2} \frac{\partial^{2} \psi}{\partial X^{2}}+\varepsilon^{2} \frac{\partial^{2} \psi}{\partial Y^{2}}\right. \\
& \left.+2 \varepsilon \frac{\partial^{2} \psi}{\partial y \partial Y}+\frac{\partial^{2} \psi}{\partial y^{2}}\right) \\
= & \varepsilon \frac{\partial \psi}{\partial X}\left(\varepsilon^{3} \frac{\partial^{3} \psi}{\partial Y \partial X^{2}}+\varepsilon^{3} \frac{\partial^{3} \psi}{\partial Y^{3}}+3 \varepsilon^{2} \frac{\partial^{3} \psi}{\partial y \partial Y^{2}}\right. \\
& \left.+3 \varepsilon \frac{\partial^{3} \psi}{\partial y^{2} \partial Y}-\varepsilon\left(\varepsilon \frac{\partial \psi}{\partial Y}+\varepsilon^{2} \frac{\partial^{3} \psi}{\partial y \partial X^{2}}+\frac{\partial^{3} \psi}{\partial y^{3}}\right)+\frac{\partial \psi}{\partial y}\right) \\
& +\left(\varepsilon^{2} \frac{\partial^{3} \psi}{\partial X^{3}}+\varepsilon^{2} \frac{\partial^{3} \psi}{\partial Y^{2} \partial X}+2 \varepsilon \frac{\partial^{3} \psi}{\partial y \partial Y \partial X}+\frac{\partial^{3} \psi}{\partial y^{2} \partial X}\right)
\end{aligned}
$$

Substituting (5)-(7) into (3) yields

$$
\begin{aligned}
& \left(\varepsilon^{3} \frac{\partial}{\partial T}-\varepsilon c_{0} \frac{\partial}{\partial X}-\varepsilon \Psi_{0 y} \frac{\partial}{\partial X}\right)\left(\varepsilon^{2} \frac{\partial^{2} \psi}{\partial X^{2}}+\varepsilon^{2} \frac{\partial^{2} \psi}{\partial Y^{2}}+2 \varepsilon \frac{\partial^{2} \psi}{\partial y \partial Y}+\frac{\partial^{2} \psi}{\partial y^{2}}\right) \\
& +\varepsilon^{3} \frac{\partial \psi}{\partial X}\left(\varepsilon^{3} \frac{\partial^{3} \psi}{\partial Y \partial X^{2}}+\varepsilon^{3} \frac{\partial^{3} \psi}{\partial Y^{3}}+3 \varepsilon^{2} \frac{\partial^{3} \psi}{\partial y \partial Y^{2}}+3 \varepsilon \frac{\partial^{3} \psi}{\partial y^{2} \partial Y}\right. \\
& \left.+\varepsilon^{2} \frac{\partial^{3} \psi}{\partial y \partial X^{2}}+\frac{\partial^{3} \psi}{\partial y^{3}}\right)-\varepsilon^{3}\left(\varepsilon \frac{\partial \psi}{\partial Y}+\frac{\partial \psi}{\partial y}\right)\left(\varepsilon^{2} \frac{\partial^{3} \psi}{\partial X^{3}}+\varepsilon^{2} \frac{\partial^{3} \psi}{\partial Y^{2} \partial X}\right. \\
& \left.+2 \varepsilon \frac{\partial^{3} \psi}{\partial y \partial Y \partial X}+\frac{\partial^{3} \psi}{\partial y^{2} \partial X}\right)+\varepsilon \lambda_{0}^{2} c_{0} \frac{\partial \psi}{\partial X}-\varepsilon^{3} \lambda_{0}^{2} \frac{\partial \psi}{\partial T} \\
& +\varepsilon\left(\beta_{0}+\Psi_{0 y y y}\right) \frac{\partial \psi}{\partial X}-\varepsilon\left(\lambda_{0}^{2}-\frac{\partial^{2}}{\partial y^{2}}\right) \Psi_{0 T}=0 .
\end{aligned}
$$

Expand the perturbation stream function $\psi$ in terms of $\varepsilon$ in the form

$$
\psi=\psi_{0}+\varepsilon \psi_{1}+\varepsilon^{2} \psi_{2}+\cdots
$$

Substituting (9) into (8), and then requiring all the coefficients of different powers of $\varepsilon$ to be zero, we obtain the following first-order equation of $\varepsilon$ :

$$
\begin{aligned}
\mathcal{E}^{1}:\left(c_{0}+\Psi_{0 y}\right) \frac{\partial}{\partial X} \frac{\partial^{2} \psi_{0}}{\partial y^{2}} \\
\quad-\left(\lambda_{0}^{2} c_{0}+\beta_{0}+\Psi_{0 y y y}\right) \frac{\partial \psi_{0}}{\partial X}=\Psi_{0 y y T}-\lambda_{0}^{2} \Psi_{0 T} .
\end{aligned}
$$

We assume that $\psi_{0}$ has the following variable separation solution:

$$
\psi_{0}=A(X, Y, T) G_{0}(y, T) .
$$


Substituting (11) into (10), we obtain

$$
\begin{gathered}
\Psi_{0 y y}=\lambda_{0}^{2} \Psi_{0}+F_{1}, \\
\left(\Psi_{0 y}+c_{0}\right) G_{0 y y}-G_{0}\left(\lambda_{0}^{2} c_{0}+\beta_{0}+F_{1 y}+\lambda_{0}^{2} \Psi_{0 y}\right)=0,
\end{gathered}
$$

where $F_{1}=F_{1}(y)$ is an arbitrary integral function of variable $y$.

In order to obtain solitary wave amplitude equation, we continue solving second-order equation about $\varepsilon$ :

$$
\begin{aligned}
\varepsilon^{2} & :\left(c_{0}+\Psi_{0 y}\right) \frac{\partial}{\partial X} \frac{\partial^{2} \psi_{1}}{\partial y^{2}}-\left(\lambda_{0}^{2} c_{0}+\beta_{0}+\Psi_{0 y y y}\right) \frac{\partial \psi_{1}}{\partial X} \\
& =-2\left(c_{0}+\Psi_{0 y}\right) \frac{\partial^{3} \psi_{0}}{\partial X \partial y \partial Y} .
\end{aligned}
$$

Then, we assume that $\psi_{1}$ has the following variable separation solution:

$$
\psi_{1}=A_{Y}(X, Y, T) G_{1}(y, T) .
$$

Substituting (15) into (14), we have

$$
\begin{aligned}
& \left(\Psi_{0 y}+c_{0}\right) G_{1 y y}-G_{1}\left(\lambda_{0}^{2} c_{0}+\beta_{0}+F_{1 y}+\lambda_{0}^{2} \Psi_{0 y}\right) \\
& \quad=-2\left(\Psi_{0 y}+c_{0}\right) G_{0 y} .
\end{aligned}
$$

The governing equation of solitary wave amplitude still cannot be obtained from (14), and we continue solving the following high-order problem:

$$
\begin{aligned}
& \varepsilon^{3}:\left(c_{0}+\Psi_{0 y}\right) \frac{\partial}{\partial X} \frac{\partial^{2} \psi_{2}}{\partial y^{2}}-\left(\lambda_{0}^{2} c_{0}+\beta_{0}+\Psi_{0 y y y}\right) \frac{\partial \psi_{2}}{\partial X} \\
&=-2\left(c_{0}+\Psi_{0 y}\right) \frac{\partial^{3} \psi_{1}}{\partial X \partial y \partial Y}+\frac{\partial}{\partial T} \frac{\partial^{2} \psi_{0}}{\partial y^{2}} \\
&-\left(c_{0}+\Psi_{0 y}\right) \frac{\partial^{3} \psi_{0}}{\partial X^{3}}-\left(c_{0}+\Psi_{0 y}\right) \frac{\partial^{3} \psi_{0}}{\partial X \partial Y^{2}}+\frac{\partial \psi_{0}}{\partial X} \frac{\partial^{3} \psi_{0}}{\partial y^{3}} \\
&-\frac{\partial \psi_{0}}{\partial y} \frac{\partial^{3} \psi_{0}}{\partial y^{2} \partial X}-\lambda_{0}^{2} \frac{\partial \psi_{0}}{\partial T} .
\end{aligned}
$$

Substituting (11), (13), and (15) into (17), the following can be obtained:

$$
\begin{aligned}
&\left(c_{0}+\Psi_{0 y}\right) \frac{\partial}{\partial X} \frac{\partial^{2} \psi_{2}}{\partial y^{2}}-\left(\lambda_{0}^{2} c_{0}+\beta_{0}+\Psi_{0 y y y}\right) \frac{\partial \psi_{2}}{\partial X} \\
&=A_{T}\left(G_{0 y y}-\lambda_{0}^{2} G_{0}\right)+\left(G_{0} G_{0 y y y}-G_{0 y} G_{0 y y}\right) A A_{X} \\
&-\left(c_{0}+\Psi_{0 y}\right) G_{0} A_{X X X}-\left(c_{0}+\Psi_{0 y}\right)\left(2 G_{1 y}+G_{0}\right) A_{X Y Y} \\
&+\left(G_{0 y y T}-\lambda_{0}^{2} G_{0 T}\right) A \\
&= \frac{F_{1 y}+\beta_{0}}{c_{0}+\Psi_{0 y}} G_{0} A_{T}+G_{0}^{2}\left(\frac{\lambda_{0}^{2} c_{0}+\lambda_{0}^{2} \Psi_{0 y}+F_{1 y}+\beta_{0}}{c_{0}+\Psi_{0 y}}\right)_{y} A A_{X} \\
&-\left(c_{0}+\Psi_{0 y}\right) G_{0} A_{X X X}-\left(c_{0}+\Psi_{0 y}\right)\left(2 G_{1 y}+G_{0}\right) A_{X Y Y} \\
&+\left(\frac{F_{1 y}+\beta_{0}}{c_{0}+\Psi_{0 y}} G_{0}\right)_{T} A
\end{aligned}
$$

$$
\begin{aligned}
= & G_{0}^{2} \frac{\left(\lambda_{0}^{2} \Psi_{0 y y}+F_{1 y y}\right)\left(c_{0}+\Psi_{0 y}\right)-\Psi_{0 y y}\left(\lambda_{0}^{2} c_{0}+\lambda_{0}^{2} \Psi_{0 y}+F_{1 y}+\beta_{0}\right)}{\left(c_{0}+\Psi_{0 y}\right)^{2}} \\
& \cdot A A_{X}+\frac{F_{1 y}+\beta_{0}}{c_{0}+\Psi_{0 y}} G_{0} A_{T}-\left(c_{0}+\Psi_{0 y}\right) G_{0} A_{X X X} \\
& -\left(c_{0}+\Psi_{0 y}\right)\left(2 G_{1 y}+G_{0}\right) A_{X Y Y} \\
& +\left[G_{0 T} \frac{F_{1 y}+\beta_{0}}{c_{0}+\Psi_{0 y}}-\frac{\Psi_{0 y T}\left(F_{1 y}+\beta\right)}{\left(c_{0}+\Psi_{0 y}\right)^{2}} G_{0}\right] A .
\end{aligned}
$$

In the derivation of many nonlinear equations, the $y$-average method is the traditional method and commonly utilized, but we remove this treatment and introduce higher order $\psi_{2}$ as

$$
\begin{aligned}
\psi_{2}= & B_{1}(y, T) A^{2}+B_{2}(y, T) A_{X X}+B_{3}(y, T) A_{Y Y} \\
& +B_{4}(y, T) X+B_{5}(y, T) \int A d X,
\end{aligned}
$$

where $B_{i}(y, T)(i=1,2, \ldots, 5)$ are arbitrary functions of variables $y$ and $T$.

Therefore, we have

$$
\begin{aligned}
\frac{\partial \psi_{2}}{\partial X}=2 B_{1} & (y, T) A A_{X}+B_{2}(y, T) A_{X X X} \\
+ & B_{3}(y, T) A_{X Y Y}+B_{4}(y, T)+B_{5}(y, T) A, \\
\frac{\partial}{\partial X} \frac{\partial^{2} \psi_{2}}{\partial y^{2}}= & 2 B_{1 y y}(y, T) A A_{X}+B_{2 y y}(y, T) A_{X X X} \\
& +B_{3 y y}(y, T) A_{X Y Y}+B_{4 y y}(y, T) \\
& +B_{5 y y}(y, T) A .
\end{aligned}
$$

Substituting (20) and (21) into (18), the following can be obtained:

$$
\begin{aligned}
A_{T}+ & {\left[G_{0}^{2} \frac{\left(\lambda_{0}^{2} \Psi_{0 y y}+F_{1 y y}\right)\left(c_{0}+\Psi_{0 y}\right)-\Psi_{0 y y}\left(\lambda_{0}^{2} c_{0}+\lambda_{0}^{2} \Psi_{0 y}+F_{1 y}+\beta_{0}\right)}{\left(c_{0}+\Psi_{0 y}\right)^{2}}\right.} \\
& \left.-2\left(c_{0}+\Psi_{0 y}\right) B_{1 y y}+2\left(\lambda_{0}^{2} c_{0}+\lambda_{0}^{2} \Psi_{0 y}+F_{1 y}+\beta_{0}\right) B_{1}\right] \\
& \cdot \frac{c_{0}+\Psi_{0 y}}{\left(F_{1 y}+\beta\right) G_{0}} A A_{X}-\left[\left(c_{0}+\Psi_{0 y}\right) G_{0}+\left(c_{0}+\Psi_{0 y}\right) B_{2 y y}\right. \\
& \left.-\left(c_{0} \lambda_{0}^{2}+\lambda_{0}^{2} \Psi_{0 y}+F_{1 y}+\beta\right) B_{2}\right] \frac{c_{0}+\Psi_{0 y}}{\left(F_{1 y}+\beta\right) G_{0}} A_{X X X} \\
& -\left[\left(c_{0}+\Psi_{0 y}\right)\left(2 G_{1 y}+G_{0}\right)+\left(c_{0}+\Psi_{0 y}\right) B_{3 y y}\right. \\
& \left.-\left(c_{0} \lambda_{0}^{2}+\lambda_{0}^{2} \Psi_{0 y}+F_{1 y}+\beta\right) B_{3}\right] \frac{c_{0}+\Psi_{0 y}}{\left(F_{1 y}+\beta\right) G_{0}} A_{X Y Y} \\
& -\left[\frac{\Psi_{0 y T}\left(F_{1 y}+\beta\right)}{\left(c_{0}+\Psi_{0 y}\right)^{2}} G_{0}+\left(c_{0}+\Psi_{0 y}\right) B_{5 y y}-G_{0 T} \frac{F_{1 y}+\beta_{0}}{c_{0}+\Psi_{0 y}}\right. \\
& \left.-\left(c_{0} \lambda_{0}^{2}+\lambda_{0}^{2} \Psi_{0 y}+F_{1 y}+\beta\right) B_{5}\right] \frac{c_{0}+\Psi_{0 y}}{\left(F_{1 y}+\beta\right) G_{0}} A \\
& +\left[\left(c_{0} \lambda_{0}^{2}+\lambda_{0}^{2} \Psi_{0 y}+F_{1 y}+\beta\right) B_{4}-\left(c_{0}+\Psi_{0 y}\right) B_{4 y y}\right] \frac{c_{0}+\Psi_{0 y}}{\left(F_{1 y}+\beta\right) G_{0}}=0,
\end{aligned}
$$


where $B_{i}=B_{i}(y, T)(i=1,2, \ldots, 5)$.

When $B_{i}(i=1,2, \ldots, 5)$ satisfy the relationships,

$$
\begin{aligned}
G_{0}^{2} & {\left[\left(\lambda_{0}^{2} \Psi_{0 y y}+F_{1 y y}\right)\left(c_{0}+\Psi_{0 y}\right)-\Psi_{0 y y}\left(\lambda_{0}^{2} c_{0}+\lambda_{0}^{2} \Psi_{0 y}+F_{1 y}+\beta_{0}\right)\right] } \\
& -2\left(c_{0}+\Psi_{0 y}\right)^{3} B_{1 y y}+2\left(c_{0}+\Psi_{0 y}\right)^{2}\left(\lambda_{0}^{2} c_{0}+\lambda_{0}^{2} \Psi_{0 y}+F_{1 y}+\beta_{0}\right) B_{1} \\
& -G_{0} e_{1}\left(c_{0}+\Psi_{0 y}\right)\left(F_{1 y}+\beta\right)=0, \\
\left(c_{0}+\Psi_{0 y}\right)\left(c_{0} \lambda_{0}^{2}+\lambda_{0}^{2} \Psi_{0 y}+F_{1 y}+\beta\right) B_{2}-\left(c_{0}+\Psi_{0 y}\right)^{2} G_{0} & \\
& -\left(c_{0}+\Psi_{0 y}\right)^{2} B_{2 y y}-G_{0} e_{2}\left(F_{1 y}+\beta\right)=0, \\
\left(c_{0}\right. & \left.+\Psi_{0 y}\right)\left(c_{0} \lambda_{0}^{2}+\lambda_{0}^{2} \Psi_{0 y}+F_{1 y}+\beta\right) B_{3}-\left(c_{0}+\Psi_{0 y}\right)^{2} B_{3 y y} \\
& -\left(c_{0}+\Psi_{0 y}\right)^{2}\left(2 G_{1 y}+G_{0}\right)-G_{0} e_{3}\left(F_{1 y}+\beta\right)=0, \\
\left(c_{0}\right. & \left.+\Psi_{0 y}\right)^{2}\left(c_{0} \lambda_{0}^{2}+\lambda_{0}^{2} \Psi_{0 y}+F_{1 y}+\beta\right) B_{5}-\left(c_{0}+\Psi_{0 y}\right)^{3} B_{5 y y} \\
& -\Psi_{0 y T}\left(F_{1 y}+\beta\right) G_{0}+G_{0 T}\left(c_{0}+\Psi_{0 y}\right)\left(F_{1 y}+\beta_{0}\right) \\
& -G_{0} e_{4}\left(c_{0}+\Psi_{0 y}\right)\left(F_{1 y}+\beta\right)=0, \\
\left(c_{0}\right. & \left.+\Psi_{0 y}\right)\left(c_{0} \lambda_{0}^{2}+\lambda_{0}^{2} \Psi_{0 y}+F_{1 y}+\beta\right) B_{4}-\left(c_{0}+\Psi_{0 y}\right)^{2} B_{4 y y} \\
& -G_{0} e_{5}\left(F_{1 y}+\beta\right)=0,
\end{aligned}
$$

a $(2+1)$-dimensional variable coefficient $\mathrm{KdV}(3 \mathrm{D} \mathrm{VCKdV})$ equation is derived as follows:

$$
A_{T}+e_{1} A_{X X X}+e_{2} A A_{X}+e_{3} A_{X Y Y}+e_{4} A+e_{5}=0
$$

where $e_{i}=e_{i}(T)(i=1,2, \ldots, 5)$ are arbitrary functions of variable $T$.

\section{Exact Solution of 3D VCKdV Equation}

It is not easy to obtain the exact analytical solution of $3 \mathrm{D}$ VCKdV equation (24) in the case that 5 variable coefficients are kept arbitrary. As we know, quite a few methods for obtaining solitary wave solution of nonlinear systems have been proposed, for instance, the hyperbolic function method [16], generalized Darboux transformation [17], and CK's direct method [15]. CK's direct method is a very simple and effective method. In this section, we are going to construct the exact analytical solution of 3D VCKdV equation by CK's direct method.

The Zakharov-Kuznetsov equation is as follows:

$$
P_{\tau}+2 P P_{\xi}+P_{\xi \xi \xi}+P_{\xi \eta \eta}=0,
$$

which is taken as a two-dimensional form of constant coefficient $\mathrm{KdV}$ equation, and it has the following solitary wave solution [18]:

$$
P(\xi, \eta, \tau)=6 K^{2} \operatorname{sech}^{2}\left(K \xi+K \eta-8 K^{3} \tau\right),
$$

where $K$ is an arbitrary constant.

According to CK's direct method, we suppose the exact analytical solution of $3 \mathrm{D} \mathrm{VCKdV}$ equation in the following form:

$$
\begin{aligned}
A= & \alpha(X, Y, T)+\beta(X, Y, T) P(\xi(X, Y, T), \\
& \eta(X, Y, T), \tau(X, Y, T)) \equiv \alpha+\beta P(\xi, \eta, \tau),
\end{aligned}
$$

where $P(\xi, \eta, \tau)$ satisfy $(25)$.
From (27), we have

$$
\begin{aligned}
A_{T}= & \alpha_{T}+\beta_{T} P+\beta P_{T} \\
A_{X}= & \alpha_{X}+\beta_{X} P+\beta P_{X} \\
A_{X X X}= & \alpha_{X X X}+\beta_{X X X} P+3 \beta_{X X} P_{X}+3 \beta_{X} P_{X X}+\beta P_{X X X} \\
A_{X Y Y}= & \alpha_{X Y Y}+\beta_{X Y Y} P+2 \beta_{X Y} P_{Y}+\beta_{X} P_{Y Y}+\beta_{Y Y} P_{X} \\
& +2 \beta_{Y} P_{X Y}+\beta P_{X Y Y}, \\
P_{T}= & P_{\xi} \xi_{T}+P_{\eta} \eta_{T}+P_{\tau} \tau_{T} \\
P_{X}= & P_{\xi} \xi_{X}+P_{\eta} \eta_{X}+P_{\tau} \tau_{X} \\
P_{Y}= & P_{\xi} \xi_{Y}+P_{\eta} \eta_{Y}+P_{\tau} \tau_{Y} \\
P_{X X}= & P_{\xi \xi} \xi_{X}^{2}+2 P_{\xi \eta} \xi_{X} \eta_{X}+2 P_{\xi \tau} \xi_{X} \tau_{X}+2 P_{\eta \tau} \eta_{X} \tau_{X} \\
& +P_{\eta \eta} \eta_{X}^{2}+P_{\tau \tau} \tau_{X}^{2}, \\
& +P_{\eta \tau}\left(\eta_{X} \tau_{Y}+\eta_{Y} \tau_{X}\right)+P_{\eta \eta} \eta_{X} \eta_{Y}+P_{\tau \tau} \tau_{X} \tau_{Y} \\
P_{X Y}= & P_{\xi \xi} \xi_{Y}^{2}+2 P_{\xi \eta} \xi_{Y} \eta_{Y}+2 P_{\xi \tau} \xi_{Y} \tau_{Y}+2 P_{\eta \tau} \eta_{Y} \tau_{Y} \\
& +P_{\eta \eta} \eta_{Y}^{2}+P_{\tau \tau} \tau_{Y}^{2}, \\
P_{Y} &
\end{aligned}
$$

$$
\begin{aligned}
P_{X X X}= & P_{\xi \xi \xi} \xi_{X}^{3}+3 P_{\xi \xi \eta} \xi_{X}^{2} \eta_{X}+3 P_{\xi \xi \tau} \xi_{X}^{2} \tau_{X}+3 P_{\xi \eta \eta} \xi_{X} \eta_{X}^{2} \\
& +6 P_{\xi \eta \tau} \xi_{X} \eta_{X} \tau_{X}+3 P_{\xi \tau \tau} \xi_{X} \tau_{X}^{2}+3 P_{\eta \tau \tau} \eta_{X} \tau_{X}^{2} \\
& +3 P_{\eta \eta \tau} \tau_{X} \eta_{X}^{2}+P_{\eta \eta \eta} \eta_{X}^{3}+P_{\tau \tau \tau} \tau_{X}^{3}
\end{aligned}
$$

$$
\begin{aligned}
P_{X Y Y}= & P_{\xi \xi \xi} \xi_{X} \xi_{Y}^{2}+P_{\xi \xi \eta}\left(2 \xi_{X} \xi_{Y} \eta_{Y}+\xi_{Y}^{2} \eta_{X}\right) \\
& +P_{\xi \xi \tau}\left(2 \xi_{X} \xi_{X} \tau_{Y}+\xi_{Y}^{2} \tau_{X}\right) \\
& +P_{\xi \eta \tau} 2\left(\xi_{Y} \eta_{X} \tau_{Y}+\xi_{Y} \eta_{Y} \tau_{X}+\xi_{X} \eta_{Y} \tau_{Y}\right) \\
& +P_{\eta \eta \eta} \eta_{X} \eta_{Y}^{2}+P_{\xi \eta \eta}\left(2 \xi_{Y} \eta_{X} \eta_{Y}+\xi_{X} \eta_{Y}^{2}\right) \\
& +P_{\xi \tau \tau}\left(2 \xi_{Y} \tau_{X} \tau_{Y}+\xi_{X} \tau_{Y}^{2}\right)+P_{\eta \tau \tau}\left(2 \eta_{Y} \tau_{X} \tau_{Y}+\eta_{X} \tau_{Y}^{2}\right) \\
& +P_{\tau \tau \tau} \tau_{X} \tau_{Y}^{2} .
\end{aligned}
$$

Substituting (27)-(39) into (24), the following can be obtained:

$$
\begin{aligned}
\gamma_{0} & +\gamma_{1} P+\gamma_{2} P_{\xi}+\gamma_{3} P_{\eta}+\gamma_{4} P_{\tau}+\gamma_{5} P P_{\xi}+\gamma_{6} P P_{\eta}+\gamma_{7} P P_{\tau} \\
& +\gamma_{8} P_{\xi \xi}+\gamma_{9} P_{\xi \eta}+\gamma_{10} P_{\xi \tau}+\gamma_{11} P_{\eta \tau}+\gamma_{12} P_{\eta \eta}+\gamma_{13} P_{\tau \tau} \\
& +\gamma_{14} P_{\xi \xi \xi}+\gamma_{15} P_{\xi \xi \eta}+\gamma_{16} P_{\xi \xi \tau}+\gamma_{17} P_{\xi \eta \eta}+\gamma_{18} P_{\xi \eta \tau} \\
& +\gamma_{19} P_{\xi \tau \tau}+\gamma_{20} P_{\eta \tau \tau}+\gamma_{21} P_{\eta \eta \tau}+\gamma_{22} P_{\eta \eta \eta}+\gamma_{23} P_{\tau \tau \tau}=0
\end{aligned}
$$


where

$$
\begin{aligned}
& \gamma_{0}=\alpha_{T}+e_{2} \alpha \alpha_{X}+e_{1} \alpha_{X X X}+e_{3} \alpha_{X Y Y}+e_{4} \alpha+e_{5} \text {, } \\
& \gamma_{1}=\beta_{T}+e_{2} \alpha \beta_{X}+e_{2} \alpha_{X} \beta+e_{2} \beta \beta_{X}+e_{1} \beta_{X X X}+e_{3} \beta_{X Y Y} \\
& +e_{4} \beta \text {, } \\
& \gamma_{2}=\beta \xi_{T}+e_{2} \alpha \beta \xi_{X}+3 e_{1} \beta_{X X} \xi_{X}+2 e_{3} \beta_{X Y} \xi_{Y}+e_{3} \beta_{Y Y} \xi_{X}, \\
& \gamma_{3}=\beta \eta_{T}+e_{2} \alpha \beta \eta_{X}+3 e_{1} \beta_{X X} \eta_{X}+2 e_{3} \beta_{X Y} \eta_{Y}+e_{3} \beta_{Y Y} \eta_{X} \text {, } \\
& \gamma_{4}=\beta \tau_{T}+e_{1} \alpha \beta \tau_{X}+3 e_{2} \beta_{X X} \tau_{X}+2 e_{3} \beta_{X Y} \tau_{Y}+e_{3} \beta_{Y Y} \tau_{X} \text {, } \\
& \gamma_{5}=e_{2} \beta^{2} \xi_{X} \text {, } \\
& \gamma_{6}=e_{2} \beta^{2} \eta_{X} \\
& \gamma_{7}=e_{2} \beta^{2} \tau_{X} \\
& \gamma_{8}=3 e_{1} \beta_{X} \xi_{X}^{2}+e_{3} \beta_{X} \xi_{Y}^{2}+2 e_{3} \beta_{Y} \xi_{X} \xi_{Y}, \\
& \gamma_{9}=3 e_{1} \beta_{X} 2 \xi_{X} \eta_{X}+e_{3} \beta_{X} 2 \xi_{Y} \eta_{Y}+2 e_{3} \beta_{Y} \xi_{X} \eta_{Y}+2 e_{3} \beta_{Y} \xi_{Y} \eta_{X} \text {, } \\
& \gamma_{10}=3 e_{1} \beta_{X} 2 \xi_{X} \tau_{X}+e_{3} \beta_{X} 2 \xi_{Y} \tau_{Y}+2 e_{3} \beta_{Y} \xi_{X} \tau_{Y}+2 e_{3} \beta_{Y} \xi_{Y} \tau_{X} \text {, } \\
& \gamma_{11}=3 e_{1} \beta_{X} 2 \eta_{X} \tau_{X}+e_{3} \beta_{X} 2 \eta_{Y} \tau_{Y}+2 e_{3} \beta_{Y} \eta_{X} \tau_{Y}+2 e_{3} \beta_{Y} \eta_{Y} \tau_{X} \text {, } \\
& \gamma_{12}=3 e_{1} \beta_{X} \eta_{X}^{2}+e_{3} \beta_{X} \eta_{Y}^{2}+2 e_{3} \beta_{Y} \eta_{X} \eta_{Y}, \\
& \gamma_{13}=3 e_{1} \beta_{X} \tau_{X}^{2}+e_{3} \beta_{X} \tau_{Y}^{2}+2 e_{3} \beta_{Y} \tau_{X} \tau_{Y} \text {, } \\
& \gamma_{14}=e_{1} \beta \xi_{X}^{3}+e_{3} \beta \xi_{X} \xi_{Y}^{2} \text {, } \\
& \gamma_{15}=3 e_{1} \beta \xi_{X}^{2} \eta_{X}+2 e_{3} \beta \xi_{X} \xi_{Y} \eta_{Y}+e_{3} \beta \xi_{Y}^{2} \eta_{X}, \\
& \gamma_{16}=3 e_{1} \beta \xi_{X}^{2} \tau_{X}+2 e_{3} \beta \xi_{X} \xi_{X} \tau_{Y}+e_{3} \beta \xi_{Y}^{2} \tau_{X}, \\
& \gamma_{17}=3 e_{1} \beta \xi_{X} \eta_{X}^{2}+2 e_{3} \beta \xi_{Y} \eta_{X} \eta_{Y}+e_{3} \beta \xi_{X} \eta_{Y}^{2} \text {, } \\
& \gamma_{18}=6 e_{1} \beta \xi_{X} \eta_{X} \tau_{X}+2 e_{3} \beta \xi_{Y} \eta_{X} \tau_{Y}+2 e_{3} \beta \xi_{Y} \eta_{Y} \tau_{X} \\
& +2 e_{3} \beta \xi_{X} \eta_{Y} \tau_{Y}, \\
& \gamma_{19}=3 e_{1} \beta \xi_{X} \tau_{X}^{2}+2 e_{3} \beta \xi_{Y} \tau_{X} \tau_{Y}+e_{3} \beta \xi_{X} \tau_{Y}^{2}, \\
& \gamma_{20}=3 e_{1} \beta \eta_{X} \tau_{X}^{2}+2 e_{3} \beta \eta_{Y} \tau_{X} \tau_{Y}+e_{3} \beta \eta_{X} \tau_{Y}^{2} \text {, } \\
& \gamma_{21}=e_{1} \beta 3 \tau_{X} \eta_{X}^{2}+2 e_{3} \beta \eta_{Y} \eta_{X} \tau_{Y}+e_{3} \beta \eta_{Y}^{2} \tau_{X}, \\
& \gamma_{22}=e_{1} \beta \eta_{X}^{3}+e_{3} \beta \eta_{X} \eta_{Y}^{2} \text {, } \\
& \gamma_{23}=e_{1} \beta \tau_{X}^{3}+e_{3} \beta \tau_{X} \tau_{Y}^{2} \text {. }
\end{aligned}
$$

Comparing the coefficients of (25) and (40), we obtain

$$
\begin{aligned}
& \alpha=C e^{-\int_{0}^{T} e_{4} d \varphi}-e^{-\int_{0}^{T} e_{4} d \varphi} \int_{0}^{T} e_{5} e^{\int_{0}^{T} e_{4} d \varphi} d \varphi, \\
& \beta=\frac{\beta_{1} e_{1}}{e_{2}}, \\
& \xi=\sqrt{\frac{\beta_{1}}{2}} X-\sqrt{\frac{\beta_{1}}{2}} \int_{0}^{T} \alpha e_{2} d \varphi, \\
& \eta=\sqrt{\frac{\beta_{1} e_{1}}{2 e_{3}}} Y, \\
& \tau=\int_{0}^{T} \frac{e_{2}}{\beta_{1} e_{1}} d \varphi,
\end{aligned}
$$

where $\beta_{1} \neq 0$ and $C$ are constants, and these variable coefficients must satisfy the following equation:

$$
e_{1} e_{2} e_{4}-e_{1 T} e_{2}+e_{1} e_{2 T}=0 .
$$

Thus, the exact analytical solution of $3 \mathrm{D}$ VCKdV equation is as follows:

$$
\begin{aligned}
A(X, Y, T)= & C e^{-\int_{0}^{T} e_{4} d \varphi}-e^{-\int_{0}^{T} e_{4} d \varphi} \int_{0}^{T} e_{5} e^{\int_{0}^{T} e_{4} d \varphi} d \varphi \\
& +6 K^{2} \frac{\beta_{1} e_{1}}{e_{2}} \operatorname{sech}^{2}\left[K \sqrt{\frac{\beta_{1}}{2}} X-K \sqrt{\frac{\beta_{1}}{2}}\right. \\
& \cdot \int_{0}^{T}\left(C e^{-\int_{0}^{T} e_{4} d \varphi}-e^{-\int_{0}^{T} e_{4} d \varphi} \int_{0}^{T} e_{5} e^{\int_{0}^{T} e_{4} d \varphi} d \varphi\right) d \varphi \\
& \left.+K \sqrt{\frac{\beta_{1} e_{1}}{2 e_{3}}} Y-8 K^{3} \int_{0}^{T} \frac{e_{2}}{\beta_{1} e_{1}} d \varphi\right]
\end{aligned}
$$

\section{Atmospheric Dipole Blocking Phenomenon}

The analytical solution of 3D VCKdV equation (44) including the three variables $X, Y$, and $T$ and five arbitrary functions can be used to describe some complex atmospheric phenomena such as atmospheric blocking.

If we assume $C=e_{4}=e_{5}=0, e_{1}=e_{2}=e_{3}=P, P \neq 0$, which is an arbitrary constant, returning the variables $x, y$, and $t$, the first-order approximation solution of basic system equation (1) can be obtained:

$$
\begin{aligned}
\psi \approx & \Psi_{0}+G_{0} A \\
= & \Psi_{0}(y, t)+G_{0}(y, t)\left\{6 K^{2} \beta_{1}\right. \\
& \left.\cdot \operatorname{sech}^{2}\left[K \sqrt{\frac{\beta_{1}}{2}} \varepsilon\left(x+y-c_{0} t\right)-\frac{8 K^{3}}{\beta_{1}} \varepsilon^{3} t\right]\right\} .
\end{aligned}
$$

When taking the basic flow $\Psi_{0}(y, t)$ as

$$
\Psi_{0}=b_{0} y+b_{1},
$$

where $b_{0}$ and $b_{1}$ are arbitrary constants, by (12) and (13), we obtain

$$
G_{0}(y, t)=R\left(\varepsilon^{3} t\right) \sin \left(\sqrt{-\frac{c_{0} \lambda_{0}^{2}+\beta_{0}}{b_{0}+c_{0}}} y\right),
$$

where $R\left(\varepsilon^{3} t\right)$ is an arbitrary function of variable $t$; here, we suppose

$$
R\left(\varepsilon^{3} t\right)=\operatorname{sech}\left[30 \varepsilon^{3}(t-9)\right]
$$

and other parameters as follows:

$$
\begin{aligned}
\lambda_{0} & =0.25, \\
\beta_{0} & =1, \\
\beta_{1} & =2, \\
b_{0} & =-5, \\
b_{1} & =8, \\
K & =5 .
\end{aligned}
$$




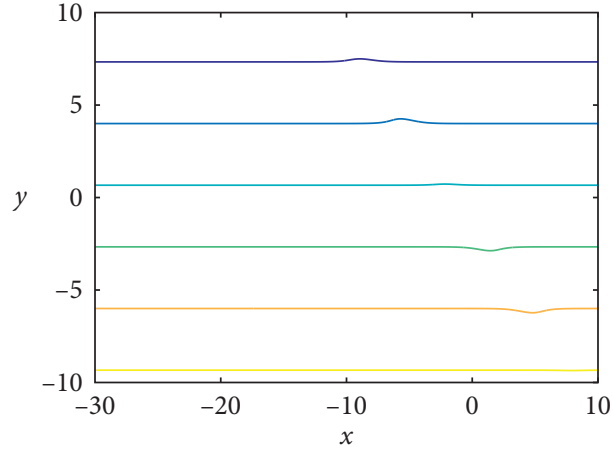

(a)

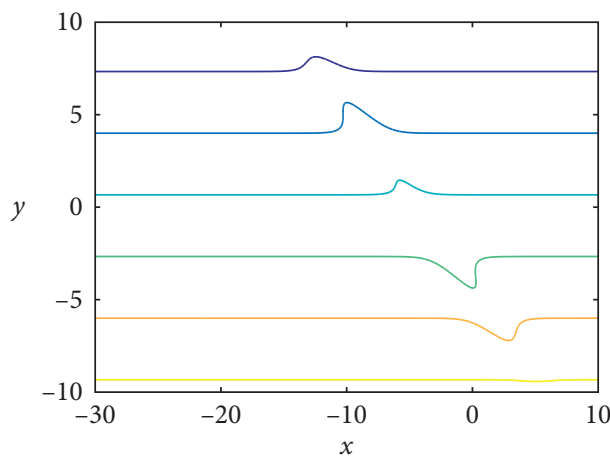

(c)

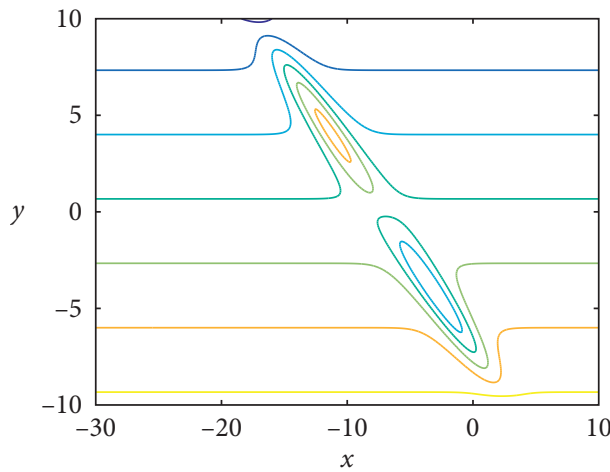

(e)

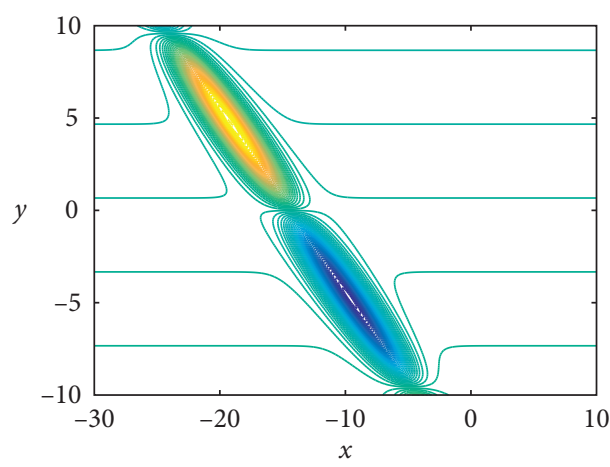

(g)

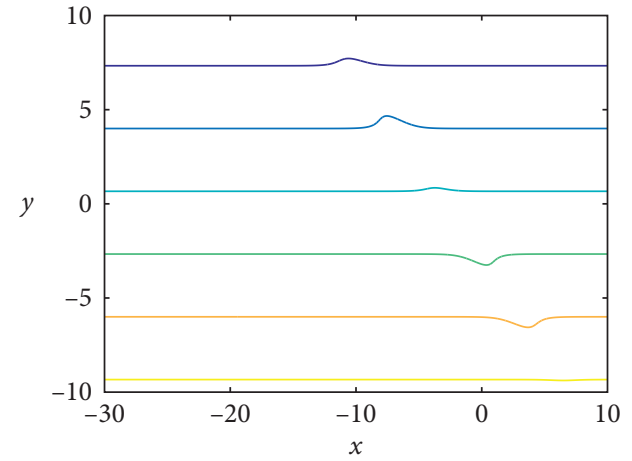

(b)

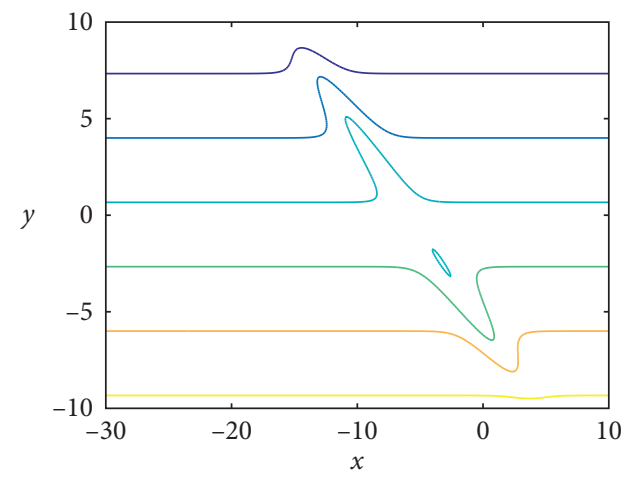

(d)

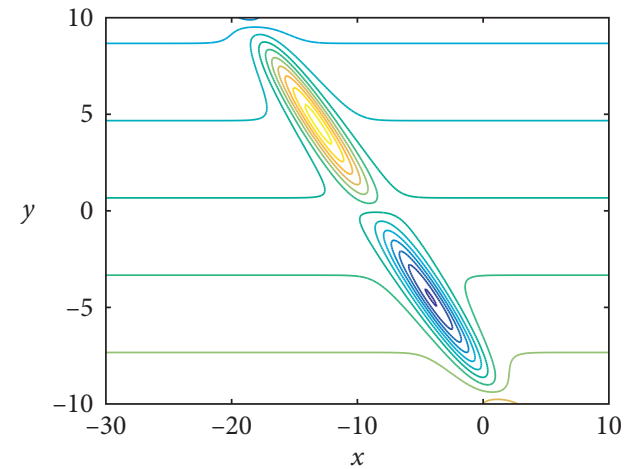

(f)

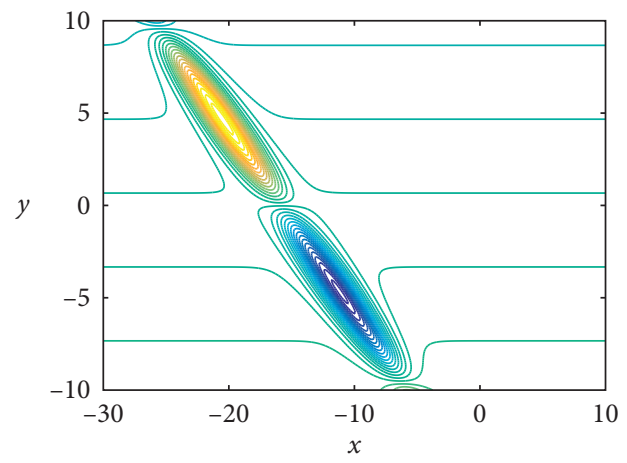

(h)

Figure 1: Continued. 


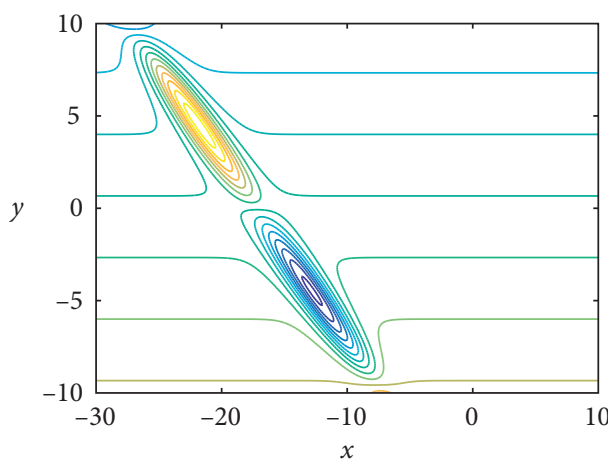

(i)

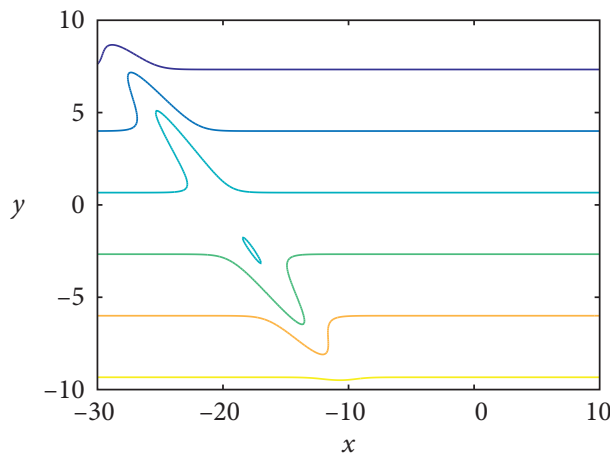

(k)

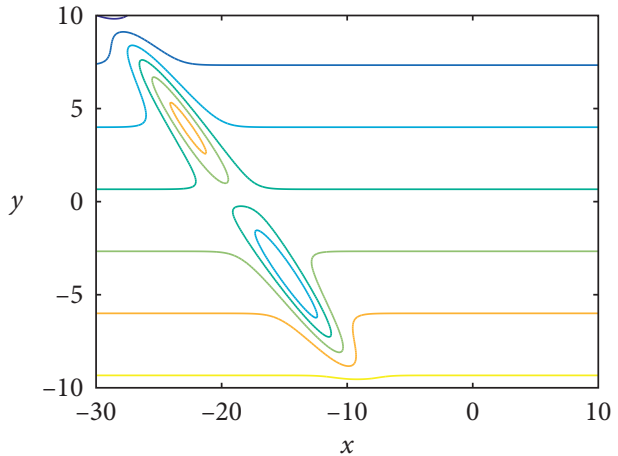

(j)

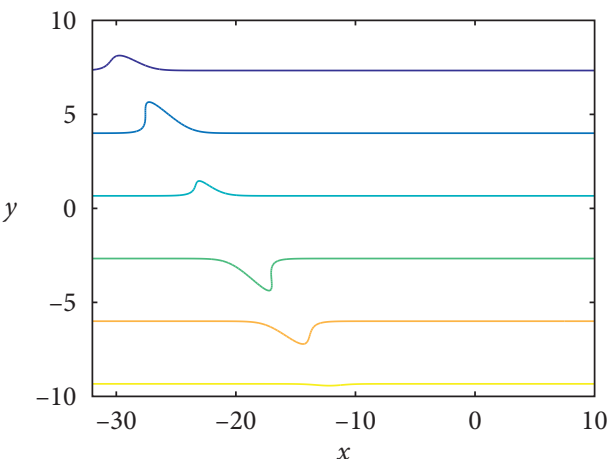

(l)

FIgURE 1: An atmospheric dipole blocking evolution with time from the theoretical solution of (45); other functions and parameters are given by (46)-(49). (a) Day 1, (b) day 2, (c) day 3, (d) day 4, (e) day 5, (f) day 6, (g) day 7, (h) day 8, (i) day 9, (j) day 10, (k) day 11, and (l) day 12 .

A dipole blocking evolution with a life cycle of twelve days is displayed in Figure 1.

Figure 1 clearly displays the onset, development, maintenance, and decay of a dipole blocking with a life cycle of twelve days. Generally speaking, the dipole blocking has a timescale of 10-20 days. Clearly, our model is able to describe such a timescale. From day 1 to day 4, the dipole blocking is at the growth status (Figures 1(a)-1(d)); at day 7 , the dipole blocking is at the strongest status (Figure 1(g)); then, it becomes weaker and finally vanishes after the eleventh day (Figures 1(h)-1(l)), and the dipole always slowly moves westward in the whole life cycle. More importantly, we can find that the central axis of the dipole is not perpendicular to the vertical direction, but it has a certain angle to the vertical direction.

Next, we make a comparison with the theoretical model VCKdV equation in [13]:

$$
A_{\tau}+e_{1} A_{\xi \xi \xi}+e_{2} A A_{\xi}+e_{3} A_{\xi}+e_{4} A+e_{5}=0 .
$$

Obviously, (50) is one-dimensional on the space model, but our derived 3D VCKdV equation (24) is twodimensional on the space.

Furthermore, [13] gives a corresponding analytical solution of (50) as

$$
\begin{aligned}
A= & -\frac{e_{3}}{e_{2}}-\sqrt{\frac{6}{\beta_{0}}} \frac{e_{1} \xi_{0}}{e_{2}}-\frac{e_{1}}{e_{2}} \int \frac{e_{1} e_{2} e_{5}-e_{1} e_{3 \tau}+e_{1 \tau} e_{3}}{e_{1}^{2}} d \tau \\
& +\frac{2 K^{2} \beta_{0} e_{1}}{e_{2}} \operatorname{sech}^{2}\left[K \sqrt{\frac{\beta_{0}}{6}} \sqrt{\varepsilon}\left(x-c_{0} t\right)+K \int e_{1} \xi_{0} d \tau\right. \\
& +\sqrt{\frac{\beta_{0}}{6}} e_{1} \int \frac{e_{1} e_{2} e_{5}-e_{1} e_{3 \tau}+e_{1 \tau} e_{3}}{e_{1}^{2}} d \tau \\
& \left.-\frac{\sqrt{6}}{9} K^{2} \beta_{0}^{3 / 2} \int e_{1} d \tau\right] .
\end{aligned}
$$

Taking $e_{3}=e_{5}=\xi_{0}=0, \quad \lambda_{0}=0.25, \quad a_{0}=-1, \quad a_{1}=3$, $\beta_{0}=1, K=8$, and $F=\operatorname{sech}\left[20 \varepsilon^{3 / 2}(t-4)\right]$, as in [13], a dipole-type blocking evolution is shown in Figure 2.

Obviously, the lifetime of dipole-type blocking in Figure 2 is eight days, and the central axis of the dipole is perpendicular to the vertical direction.

On the other hand, in order to enhance practical significance of our work, we take the Urals dipole blocking case happened from 25 January 1986 to 5 February 1986 as 


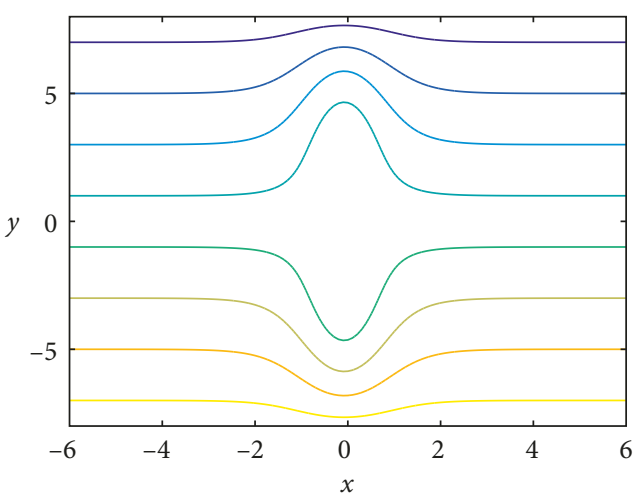

(a)

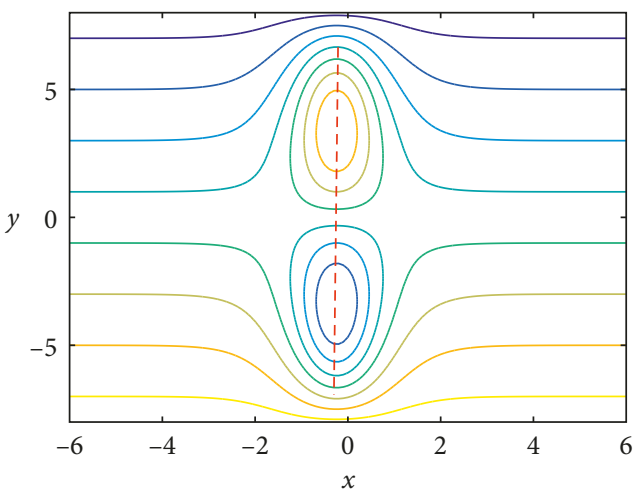

(c)

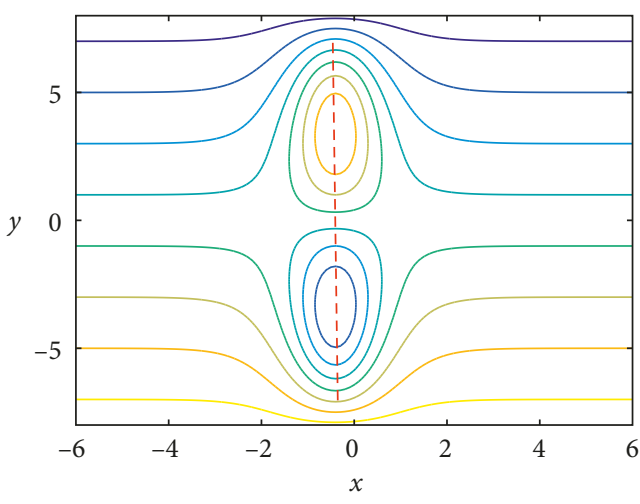

(e)

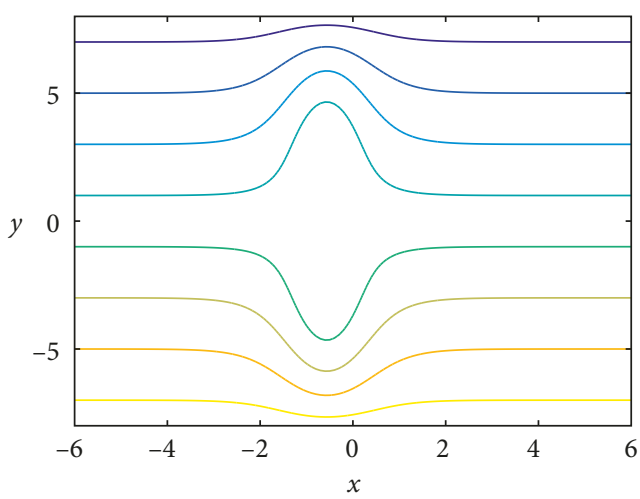

(g)

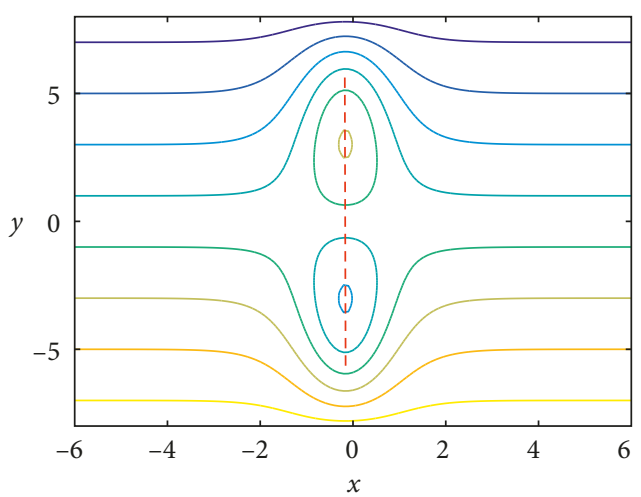

(b)

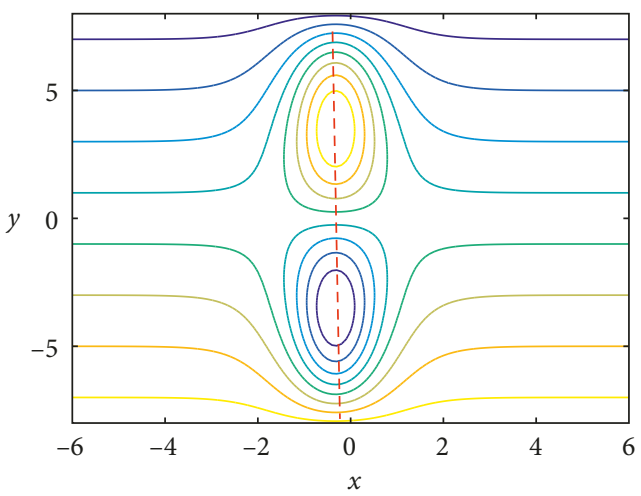

(d)

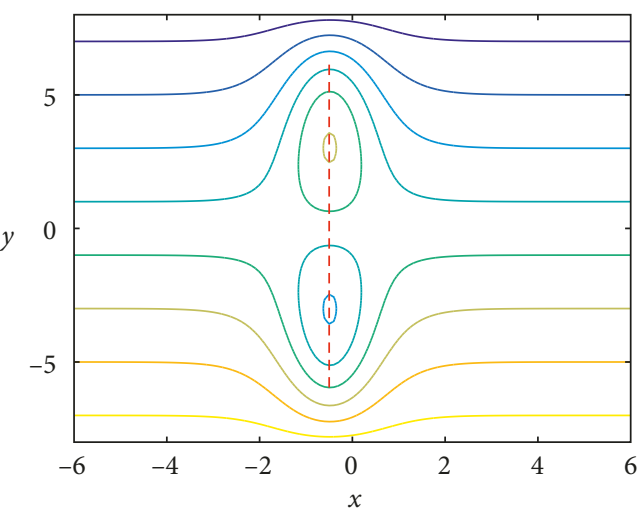

(f)

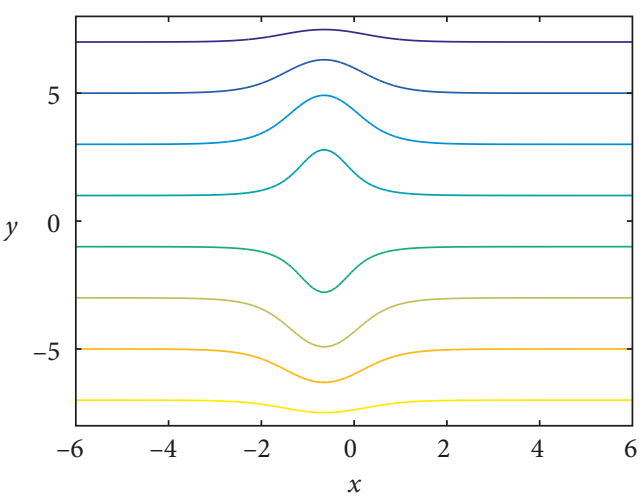

(h)

FIGURE 2: A dipole blocking life cycle from the theoretical solution of Equation (114) in [13]. (a) Day 1, (b) day 2, (c) day 3, (d) day 4, (e) day 5 , (f) day $6,(\mathrm{~g})$ day 7 , and (h) day 8. 


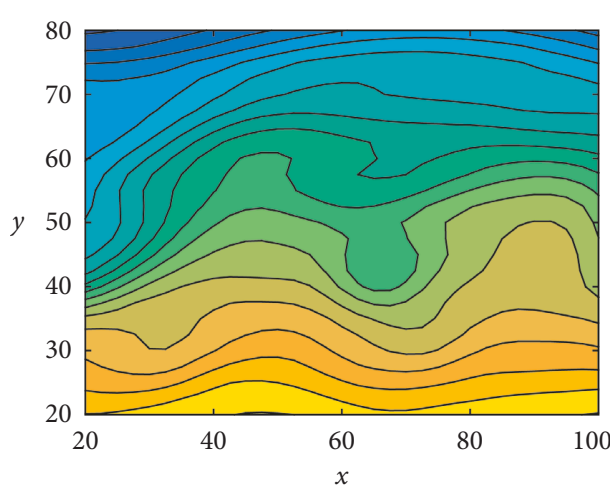

(a)

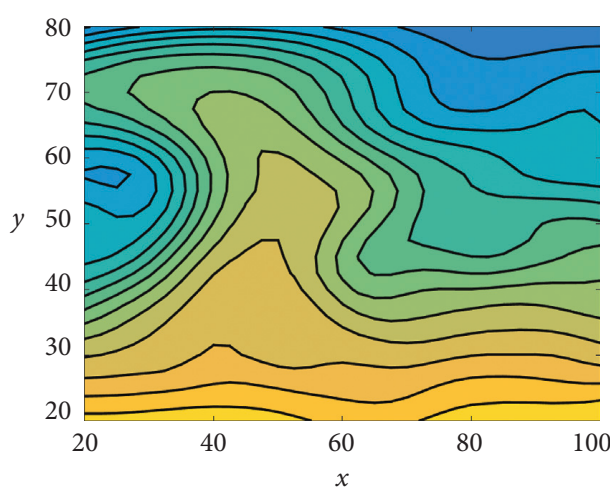

(c)

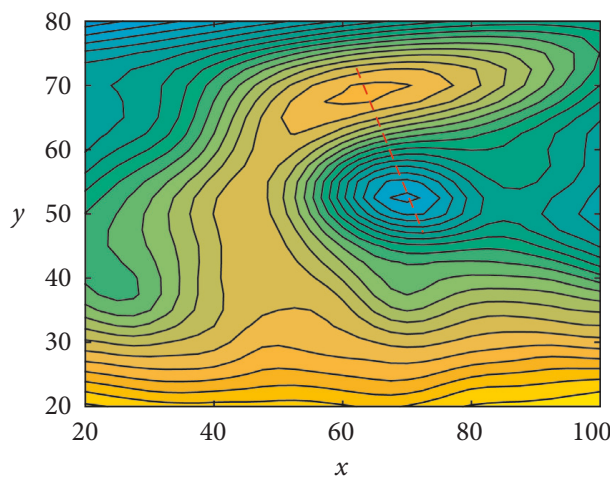

(e)

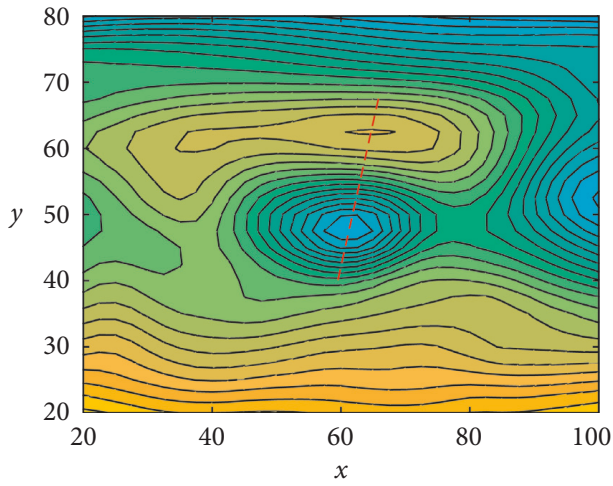

(g)

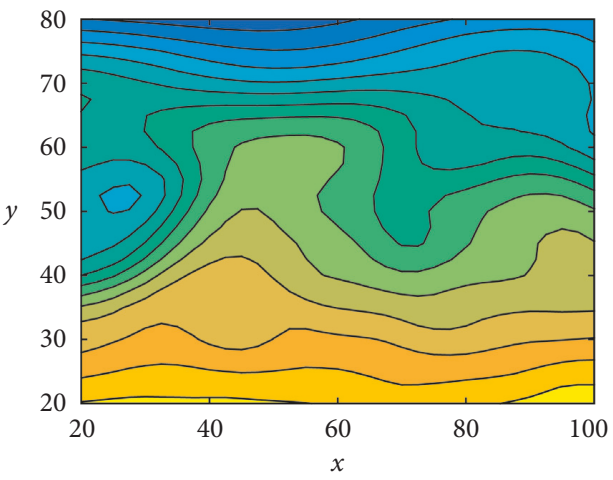

(b)

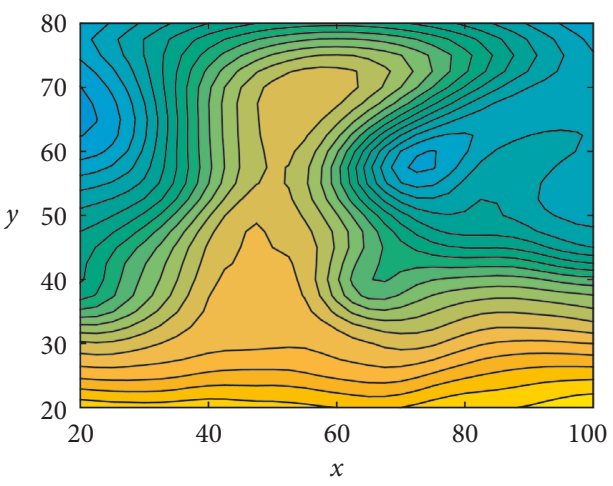

(d)

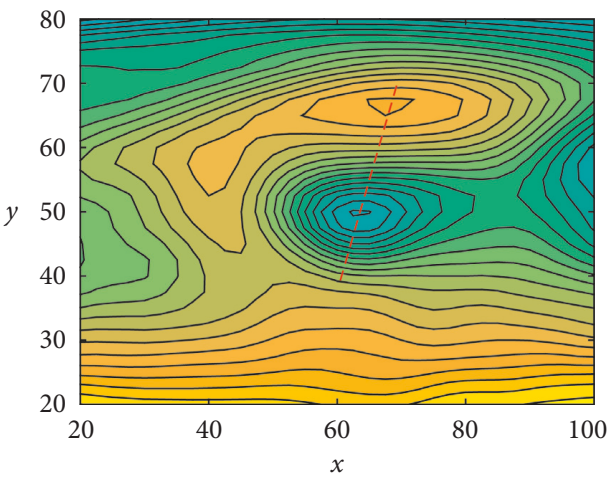

(f)

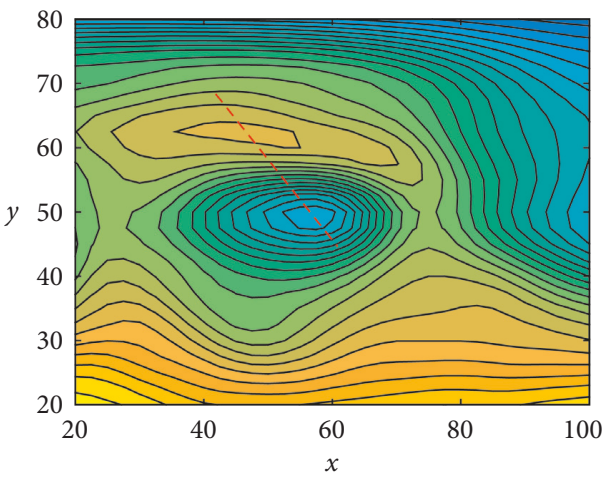

(h)

Figure 3: Continued. 


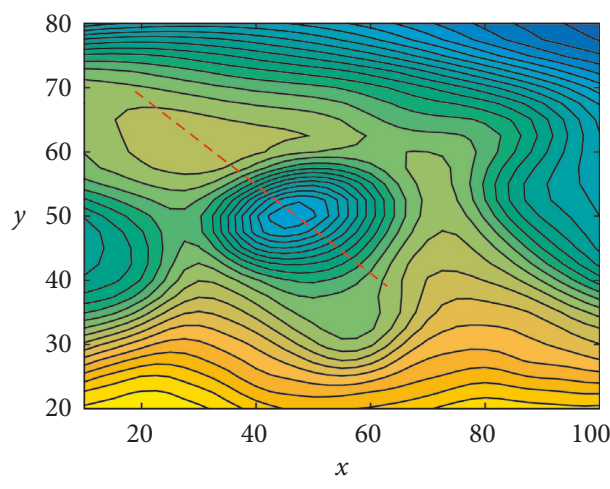

(i)

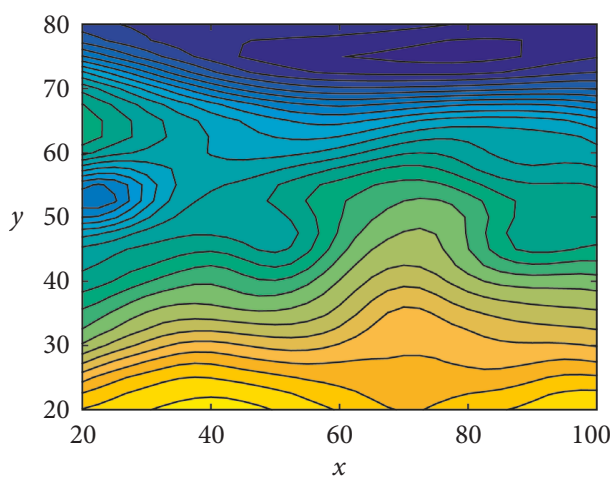

$(\mathrm{k})$

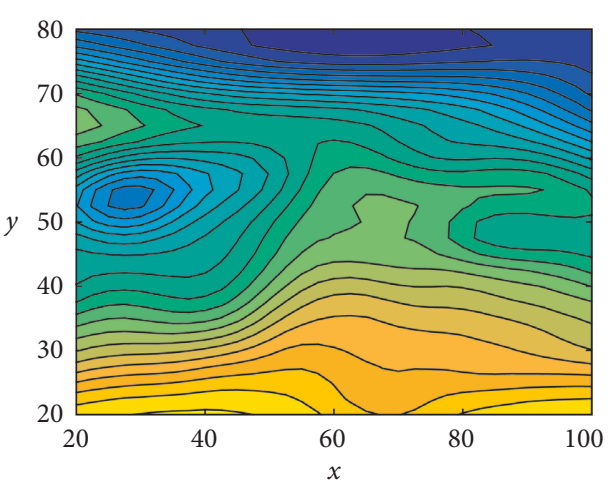

(j)

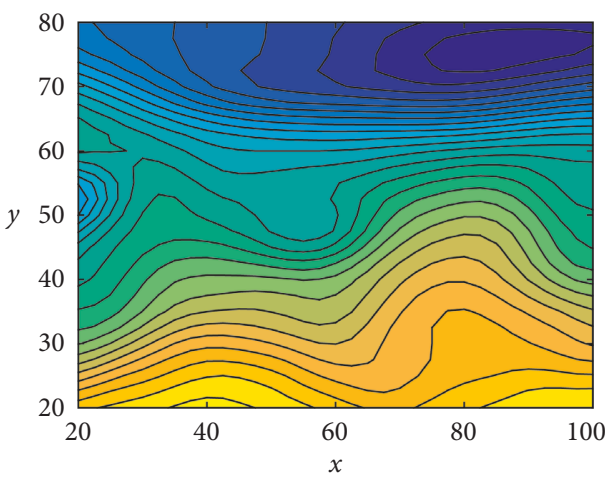

(l)

Figure 3: Geopotential height at the $500 \mathrm{hPa}$ pressure level of the Urals dipole blocking case from 25 January 1986 to 5 February 1986 . The $x$ axis is the longitude, and the $y$ axis is the latitude. (a) 25 January, (b) 26 January, (c) 27 January, (d) 28 January, (e) 29 January, (f) 30 January, (g) 31 January, (h) 1 February, (i) 2 February, (j) 3 February, (k) 4 February, and (l) 5 February.

a simple example. Making use of the National Centers for Environmental Prediction/National Center for Atmospheric Research (NCEP/NCAR) reanalysis data, the dipole blocking event is depicted in Figure 3.

It can be found from Figure 3 that there is a strong dipole blocking around Urals $\left(67^{\circ} \mathrm{N}, 66^{\circ} \mathrm{E}\right)$ with the lifetime of twelve days, and the dipole moves slowly westward. More importantly, the central axis of the dipole is not perpendicular to the vertical direction, but it has a certain angle to the vertical direction. In previous researches $[5,10,12,13]$, the central axis of the dipole is always perpendicular to the vertical direction. Making a comparison to Figures 1-3, Figure 1 is more similar to Figure 3. Consequently, our derived model is more suitable for describing the complex atmospheric blocking phenomenon.

\section{Conclusions}

In this paper, by making use of perturbation expansions and stretching transformation of a two-dimensional time and space method, a $3 \mathrm{D} \mathrm{VCKdV}$ equation is derived. This equation includes three variables and 5 arbitrary functions, and its exact analytical solution still keeps 5 arbitrary variable coefficients. Under the influence of the zonal variable $y$, the dipole blocking depicted by exact analytical solution has a certain angle to the vertical direction. As a result, our derived model is more suitable for describing the complex atmospheric blocking phenomenon compared to the previous result and real observation.

\section{Data Availability}

The data used to support the findings of this study are available from the corresponding author upon request.

\section{Conflicts of Interest}

The authors declare that there are no conflicts of interest regarding the publication of this paper and regarding the funding that the authors have received.

\section{Acknowledgments}

This work was supported by the National Natural Science Foundation of China (61673222), the Major Project of Nature Science Foundation of Higher Education Institution of Jiangsu Province of China (13KJA510001), the Research Innovation Program for College Graduates of Jiangsu Province of China (KYLX15_0873), and the Natural Science 
Research Project of Education Department of Anhui Province under Grant no. KJ2017A368.

\section{References}

[1] R. R. Long, "Solitary waves in the westerlies," Journal of the Atmospheric Sciences, vol. 21, no. 2, pp. 197-200, 1964.

[2] P. Malguzzi and P. Malanotte-Rizzoli, "Nonlinear stationary Rossby waves on nonuniform zonal winds and atmospheric blocking. Part I: the analytical theory," Journal of the Atmospheric Sciences, vol. 41, no. 17, pp. 2620-2628, 1984.

[3] P. Malanotte-Rizzoli and P. Malguzzi, "Coherent structures in a baroclinic atmosphere. Part III: block formation and eddy forcing," Journal of the Atmospheric Sciences, vol. 44, no. 17, pp. 2493-2505, 1987.

[4] G. Gottwald and R. Grimshaw, "The effect of topography on the dynamics of interacting solitary waves," Journal of the Atmospheric Sciences, vol. 56, no. 21, pp. 3663-3678, 1999.

[5] D. Luo and L. Ji, "A theory for atmospheric blocking formation in the atmosphere," Science in China (Series B), vol. 33, no. 3, pp. 69-79, 1989, in Chinese.

[6] D. Luo, F. Huang, and Y. Diao, "Interaction between antecedent planetary-scale envelope soliton blocking anticyclone and synoptic-scale eddies: observations and theory," Journal of Geophysical Research Atmospheres, vol. 106, no. D23, pp. 31795-31815, 2001.

[7] D. Luo, "A barotropic envelope Rossby soliton model for block-eddy interaction. Part I: effect of topography," Journal of the Atmospheric Sciences, vol. 62, no. 1, pp. 5-21, 2005.

[8] D. Luo, J. Cha, L. Zhong, and A. Dai, "A nonlinear multiscale interaction model for atmospheric blocking: the eddyblocking matching mechanism," Quarterly Journal of the Royal Meteorological Society, vol. 140, no. 683, pp. 1785-1808, 2014.

[9] F. Huang, X. Tang, S. Lou, and C. Lu, "Evolution of dipoletype blocking life cycles: analytical diagnoses and observations," Journal of the Atmospheric Sciences, vol. 64, no. 1, pp. 52-73, 2007.

[10] H. Yang, D. Yang, Y. Shi, S. Jin, and B. Yin, "Interaction of algebraic Rossby solitary waves with topography and atmospheric blocking," Dynamics of Atmospheres and Oceans, vol. 71, pp. 21-34, 2015.

[11] S. Y. Lou, B. Tong, H. C. Hu, and X. Y. Tang, "Coupled KdV equations derived from two-layer fluids," Journal of Physics A Mathematical and General, vol. 39, no. 3, pp. 513-527, 2006.

[12] R. C. Cascava, "Variable coefficient Korteweg-de Vries equations and wave propagation in elastic tubes," in Evolution Equations, Lecture Notes in Pure and Applied Math, vol. 234, pp. 57-69, Marcel Dekker, New York, NY, USA, 2003.

[13] T. Xiao-Yan, G. Yuan, H. Fei, and L. Sen-Yue, "Variable coefficient nonlinear systems derived from an atmospheric dynamical system," Chinese Physics B, vol. 18, no. 11, pp. 4622-4635, 2009.

[14] T. Xiao-Yan, H. Fei, and L. Sen-Yue, "Variable coefficient $\mathrm{KdV}$ equation and the analytical diagnoses of a dipole blocking life cycle," Chinese Physics Letter, vol. 23, no. 4, pp. 887-890, 2006.

[15] G. Yuan and T. Xiao-Yan, "A coupled variable coefficient modified KdV equation arising from a two-layer fluid system," Communications in Theoretical Physics, vol. 48, no. 6, pp. 961-970, 2007.

[16] H. C. Ma, D. D. Yao, and X. F. Peng, "Exact solutions of nonlinear fractional partial differential equations by fractional sub-equation method," Thermal Science, vol. 19, no. 4, pp. 1239-1244, 2015.

[17] G. Zhang and Z. Yan, "Multi-rational and semi-rational solitons and interactions for the nonlocal coupled nonlinear Schrödinger equations," EPL, vol. 118, no. 6, p. 60004, 2017.

[18] A. M. Wazwaz, "Exact solutions with solitons and periodic structures for the Zakharov-Kuznetsov (ZK) equation and its modified form," Communications in Nonlinear Science and Numerical Simulation, vol. 10, no. 6, pp. 597-606, 2005. 

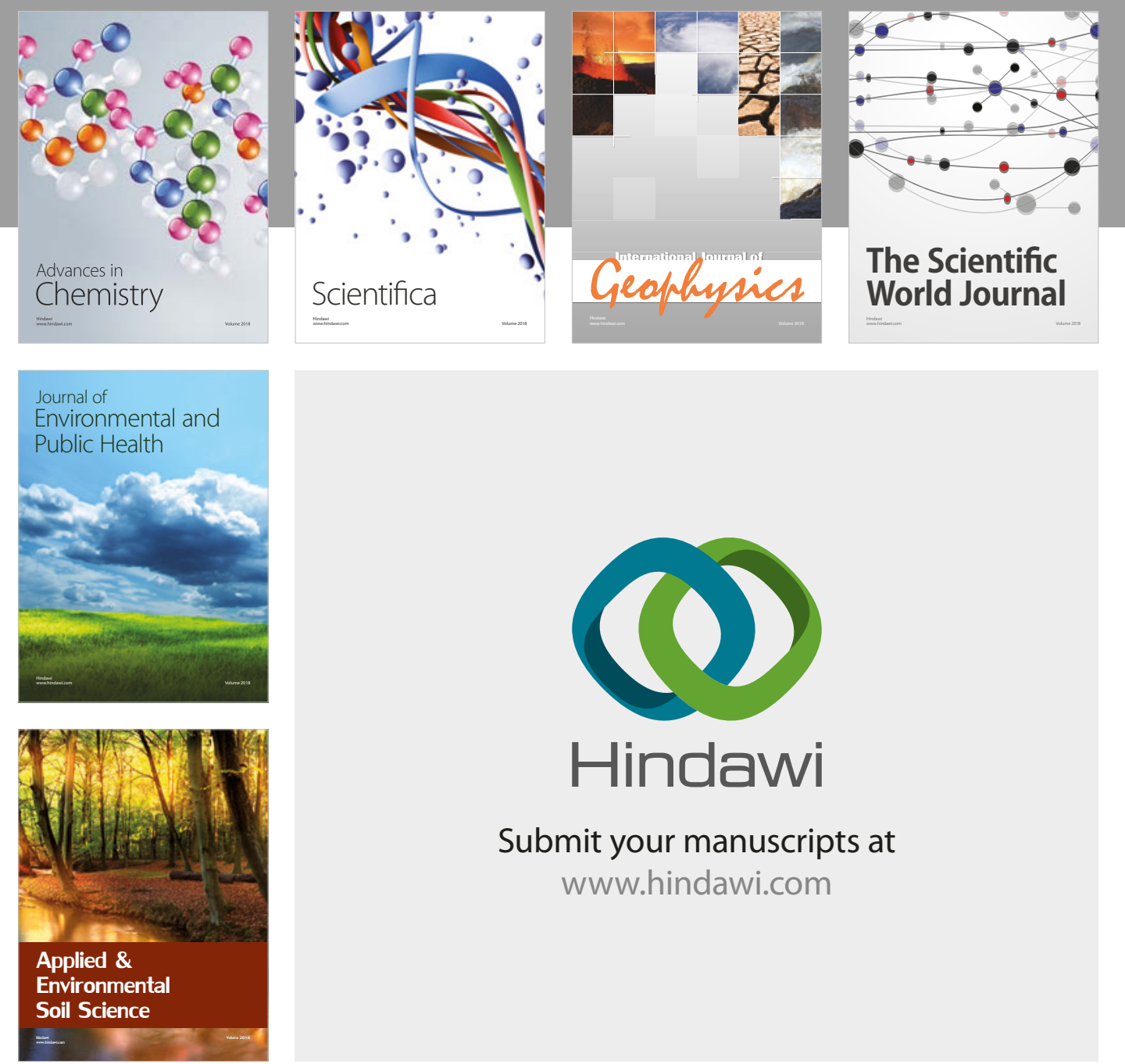

The Scientific

\section{World Journal}
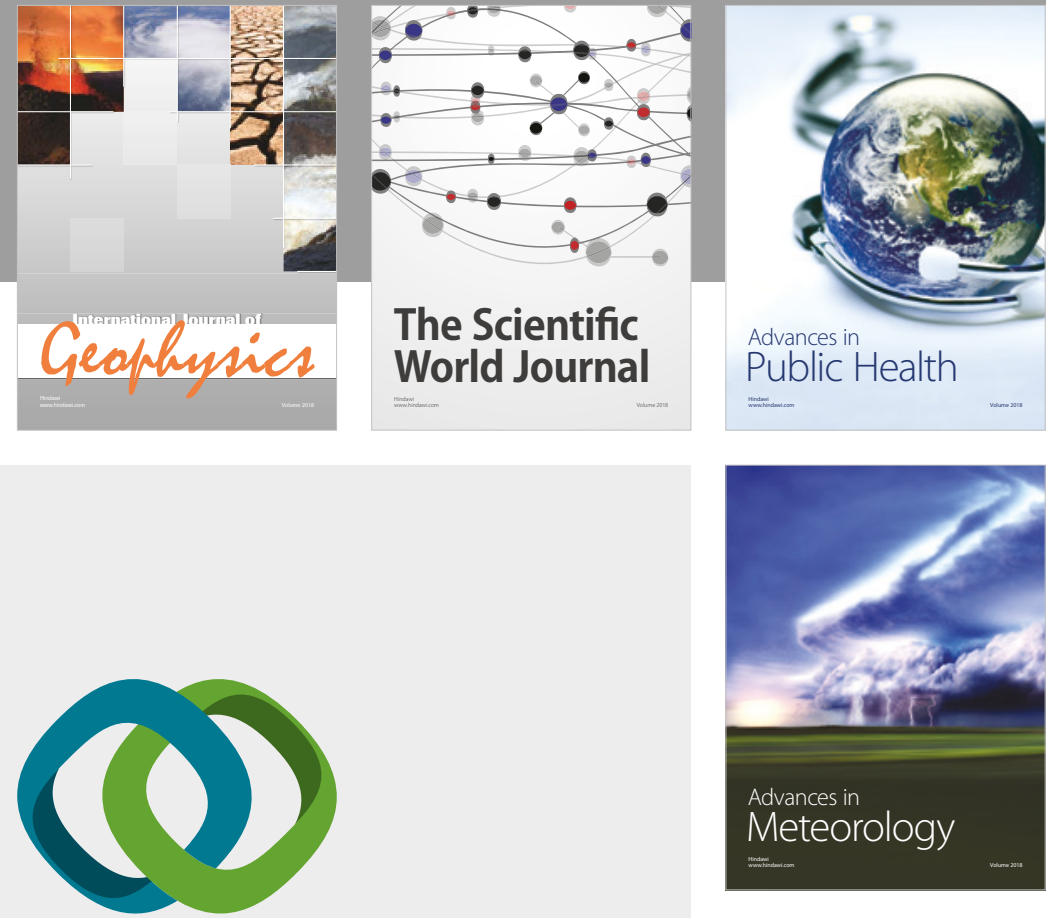

Advan

Public Health

\section{Hindawi}

Submit your manuscripts at

www.hindawi.com
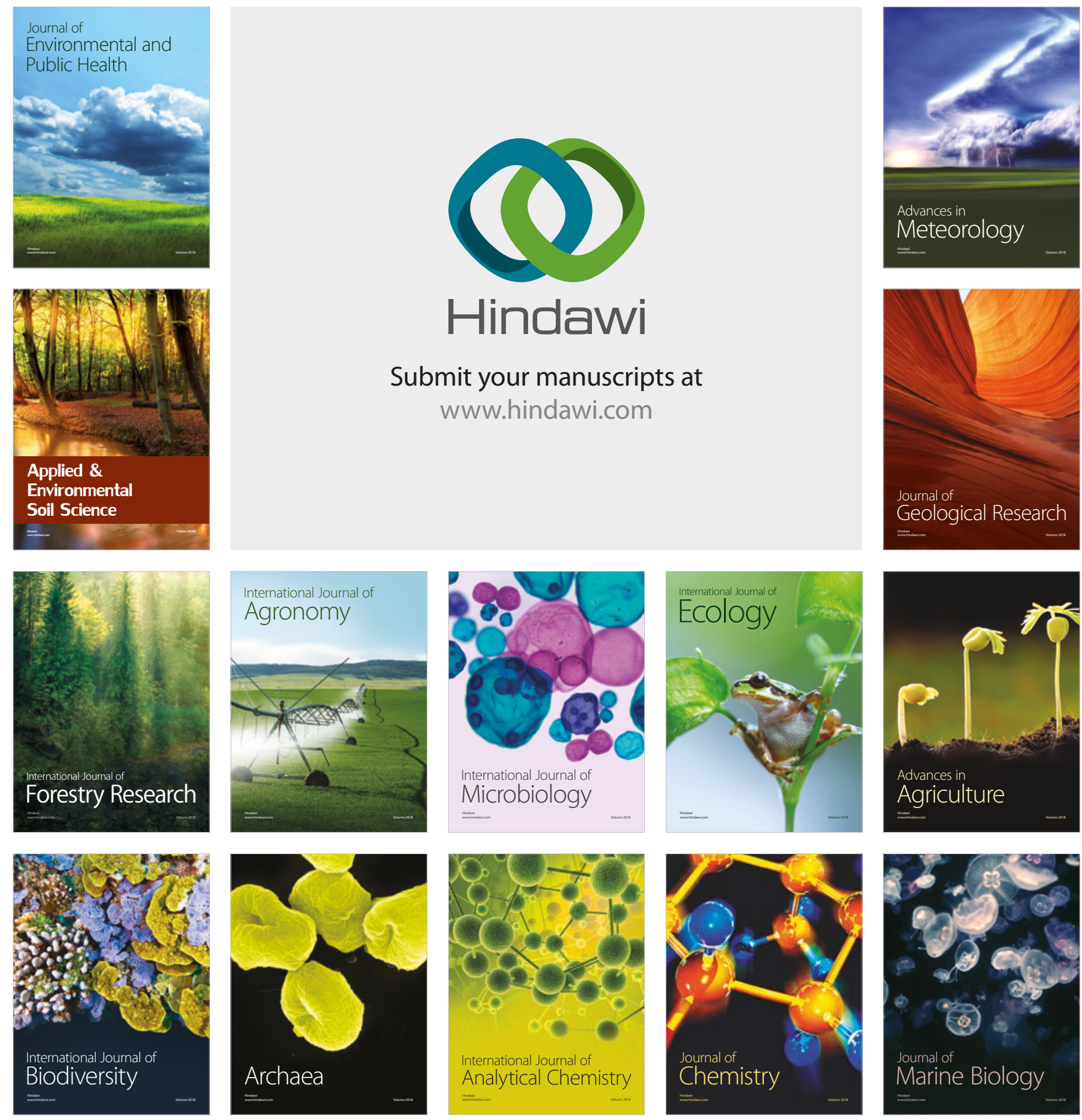